\title{
A Pilot Study for Gender Variation and Correlation of Anthropometric Parameters on the Variables of Brainstem Auditory Evoked Potentials in Audiometrically Normal Young Adults
}

\author{
Anupinder Thind', Ravdeep Singh², Avnish Kumar ${ }^{3}$ and Puneet Gambhir ${ }^{4^{*}}$ \\ 1,3Department of Physiology, Government Medical College and Rajindra Hospital (GMC\&RH) \\ Patiala - 147001, Punjab, India \\ 2Department of Forensic Medicine, Guru Gobind Singh Medical College, \\ Faridkot - 151203, Punjab, India \\ ${ }^{4}$ Department of Community, Government Medical College and Rajindra Hospital (GMC\&RH) \\ Patiala - 147001, Punjab, India; drpuneetgmc@gmail.com
}

\begin{abstract}
Head size gender and BMI have found to have correlation with Brainstem Auditory Evoked Potentials (BAEPs) in some studies but other have expressed doubts. There is no common consensus. To add to the complexity, the range of normative values also vary. The present pilot study was done aiming to study effect of gender and anthropometric parameters on the variables of BAEPs in audiometrically normal young adults and collect normative values for further studies. This was an observational prospective, cross sectional, pilot study conducted among 100 medical students with 50 males and 50 females. The selected students fulfilled the inclusion and exclusion criteria and had given informed consent for the study. In the study RMS EMG EP MARK II machine was used to measure and record the absolute, interpeak and wave amplitudes. The data was collected and statistically analysed. The BAEP measurements from the subjects were compared with gender and anthropometric measurements. The age of students enrolled in subject ranged from 18-21 years. The difference between Left and Right ears Wave I, III and V, besides I-Ia and V-Va difference was found to be significant; while differences between male and female peak latencies of Wave III (left and right) and V (left and right) and Wave IV right ear, I-V IPL and V-Va of Right ear were found to be statistically significant. BMI did not show any statistically significant correlation with BAEPs. AEP results were definitely individual effected by gender, with latency duration more among males than females in most BAERs but were significant in few. The head circumference of a person appears to affect the BAER. BMI in the study was not significant with BAER variables.
\end{abstract}

Keywords: Brainstem Auditory Evoked Potentials (BAEPs), Gender, Anthropometric, Young Adults

\section{Introduction}

Brainstem Auditory Evoked Potentials (BAEPs) or Brainstem Auditory Evoked Response (BAERs) are neural originated electrical pulses which arise within $10 \mathrm{~ms}$ after a transient acoustic stimulus (Figure 1) ${ }^{1}$. The concept of BAERs was first proposed by Jewett et al. (1970) to describe a set of 5-7 vertex positive waves evoked by acoustic stimuli which originated from the brainstem ${ }^{2}$. Clinically, it has been used to identify the dysfunction

${ }^{*}$ Author for correspondence 
of the auditory pathways especially the auditory nerve and brainstem and used in audiology, neurology, neonatology and anesthesiology $y^{3}$. Research studies on these physiology-based studies have implicated subject based factors (including age and gender) which might have a role on BAERs if stimulus, recording parameters are standardized or universal guidelines are present $t^{4}$.

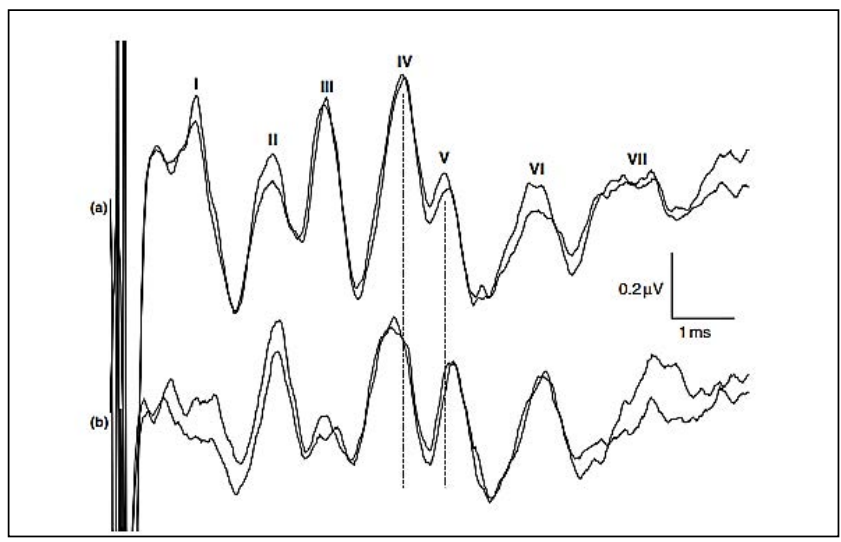

Figure 1. Normal BAEPs to monaural stimulation, (a) Vertex to ipsilateral ear lobe waveforms, with vertex-positive peaks shown as upward deflections (b) Vertex to contralateral ear lobe waveforms. Note that wave I is absent and the peak latency of wave IV has decreased, whereas that of wave V has increased (dotted lines) ${ }^{1}$.

Women have shown a greater sensitivity to hearing while differences in behavioral, morphological and physiological exist throughout the auditory system with males. These changes are likely to affect the nerve conduction.

However, lack of common consensus among studies makes the clinical role of BAERs uncertain. The availability of advanced neuroimaging techniques (MRI and CT) which can pinpoint the lesions in the auditory nerve and brainstem have posed questions on the clinical relevance of BAERs ${ }^{5,6}$. In a developing like India, the use of BAERs for the screening and diagnosis of diseases of auditory pathways provides a better and a relatively cheaper alternative as compared to costlier imaging techniques like MRI and CT. Hence, studies are required to fill in the paucity of data in the Indian context ${ }^{4}$ so as to establish BAERs as the diagnostic modality.

The present pilot study was aimed to study the influence of gender and anthropometric parameters on the variables of brainstem auditory evoked potentials in audiometrically normal young adults (As per Cambridge
Dictionary Young adult "a person who is in his or her late teenage years or early twenties"; for the present study young adult taken as age group 18 years to 22 years).

\section{Materials and Methods}

This study was conducted in the Physiology Department, GMC \& RH, Patiala after due approval by the institutional ethics committee. The study is a Prospective, Cross sectional, Observational Pilot Study conducted over a period of 1 year among Medical MBBS Students of phase I and II. Inclusion criteria included age between 18 to 22 years, normal hearing tests (In this study Rinne's test and Weber's test were used) and exclusion of history as mentioned in exclusion criteria; while the Subjects excluded were who had age $<18$ years or more than 22 years, had a history of head/ear trauma, intake of known ototoxic drugs (e.g., aminoglycosides) or any other medication that might affect normal functioning of the nervous system (e.g., antidepressants, antipsychotics, methyldopa, etc.), family history of deafness or any systemic illness that might affect the nervous system (uremia, diabetes mellitus, stroke, hepatic encephalopathy, multiple sclerosis, thyroid disorders, anemia, meningitis, etc.), history of tobacco chewing, chronic alcoholism or cigarette smoking, an year surgery, radiotherapy or chemotherapy. Besides included abnormal hearing tests (Rinne's test and Weber's test) and hearing loss (conductive as well as sensorineural) due to any cause.

The sample size (being a pilot study) was purposively decided to be $25 \%$ of the total population. i.e.; $25 \%$ of 400 students is 100 . Since the \% of the male and female students is approximately $45-49 \%$ and $51-55 \%$ respectively; the sample was equally divided into 50 each for male and female group.

Line list of all the students were prepared for males and females separately. Since approximately 200 female and 200 male students are there every $4^{\text {th }}$ student in the male and female list was enrolled after the first student being selected randomly. In case of ineligibility or no consent the next student in the list was enrolled. Informed consent was taken from all the enrolled subjects after explaining procedure, purpose and other aspects of the study in their native language.

After initial screening, the participants who fulfilled the selection criteria were enrolled in the study. Before starting the test, following were recorded 
- Age (in years rounded to nearest year)

- Height while standing with no shoes (in $\mathrm{cm}$ ),

- Body weight (in $\mathrm{kg}$ ) with minimal clothing

- Head circumference (in $\mathrm{cm}$ )

- Ear to ear Distance (in cm).

- Inion to Nasion (IN) distance (in $\mathrm{cm}$ )

The recording machine used in the study was 'RMS EMG EP MARK II' of Recorders and Medicare. As per protocol recordings were done in a semi-dark room to decrease visual excitement and with quiet surroundings to reduce audio excitement. The participants were made to sit relaxed with their back towards the recording machine after ensuring all the metallic ornaments worn by subject were removed. The participants were explained the details of the procedure. To reduce the impedance, the skin was cleaned thoroughly before the placement of electrodes. The electrodes were placed on the mastoid processes bilaterally (reference), forehead (ground) and vertex (active) ${ }^{8}$. The variables of BAER included waves I to $\mathrm{V}$ (in milliseconds), amplitude (millivolts) and interpeak latency (IPL in milliseconds) measured as the distance between the peak of two waves e. g. I-V, I-III and III-V2 ${ }^{9}$. The BAER values were obtained in the form of numerical and graphical data for each participant.

Study Parameters include Anthropometric measures of height, weight, BMI, Ear to Ear distance, Inion to Nasion (IN) distance, head circumference besides gender and BAER parameters. The subjects were considered as one unit and right and left ears were considered pairs when gender differentiation was not made.

Table 1. Demographic and anthropometric data of the study participants

\begin{tabular}{|c|c|c|c|c|c|c|c|c|c|c|c|}
\hline Parameters & Obs & Mean & $\begin{array}{l}\text { Std } \\
\text { Dev }\end{array}$ & Min & $25 \%$ & Median & $75 \%$ & $\operatorname{Max}$ & Mode & Test $^{*}$ & p-value \\
\hline \multicolumn{12}{|c|}{ Age (In years) } \\
\hline Overall & 100 & 18.64 & 0.77 & 18 & 18 & 18 & 19 & 21 & 18 & & \\
\hline Females & 50 & 18.54 & 0.65 & 18 & 18 & 18 & 19 & 20 & 18 & \multirow{2}{*}{$\begin{array}{c}\text { Mann- } \\
\text { Whitney } \\
\text { test }\end{array}$} & \multirow{2}{*}{$p=0.367$} \\
\hline Males & 50 & 18.74 & 0.88 & 18 & 18 & 18.5 & 19 & 21 & 18 & & \\
\hline \multicolumn{12}{|c|}{ Height (in m) } \\
\hline Overall & 100 & 1.66 & 0.09 & 1.47 & 1.59 & 1.65 & 1.72 & 1.95 & 1.64 & & \\
\hline Females & 50 & 1.59 & 0.06 & 1.47 & 1.53 & 1.59 & 1.64 & 1.75 & 1.52 & \multirow{2}{*}{ t test } & \multirow{2}{*}{$<0.001$} \\
\hline Males & 50 & 1.72 & 0.07 & 1.55 & 1.67 & 1.72 & 1.76 & 1.95 & 1.7 & & \\
\hline \multicolumn{12}{|c|}{ Weight (in Kg) } \\
\hline Overall & 100 & 60.3 & 13.0 & 35 & 50 & 59 & 68 & 110 & 52 & & \\
\hline Females & 50 & 52.2 & 7.3 & 35 & 47 & 51.5 & 57 & 75 & 52 & \multirow{2}{*}{$\begin{array}{c}\text { Mann- } \\
\text { Whitney } \\
\text { test }\end{array}$} & \multirow[b]{2}{*}{$<0.001$} \\
\hline Males & 50 & 68.4 & 12.3 & 48 & 61 & 65.5 & 78 & 110 & 63 & & \\
\hline \multicolumn{12}{|l|}{$\mathrm{BMI}\left(\mathrm{Kg} / \mathbf{m}^{2}\right)$} \\
\hline Overall & 100 & 21.8 & 3.3 & 16.2 & 19.5 & 21.5 & 24.2 & 32.8 & 22.6 & & \\
\hline Females & 50 & 20.7 & 2.9 & 16.2 & 18.5 & 20.5 & 21.9 & 30.0 & 18.4 & \multirow{2}{*}{$\begin{array}{c}\text { Mann- } \\
\text { Whitney } \\
\text { test }\end{array}$} & \multirow{2}{*}{0.0003} \\
\hline Males & 50 & 23.0 & 3.3 & 16.8 & 20.8 & 22.6 & 25.1 & 32.8 & 22.6 & & \\
\hline \multicolumn{12}{|c|}{ Head circumference (in $\mathrm{cm}$ ) } \\
\hline Overall & 100 & 55.5 & 1.8 & 52.5 & 54 & 55.3 & 57 & 60 & 55 & & \\
\hline Females & 50 & 54.5 & 1.5 & 52.5 & 53.5 & 54.3 & 55 & 59 & 55 & \multirow{2}{*}{$\begin{array}{c}\text { Mann- } \\
\text { Whitney } \\
\text { test }\end{array}$} & \multirow{2}{*}{$<0.001$} \\
\hline Males & 50 & 56.5 & 1.4 & 53.5 & 55.5 & 56.5 & 57.5 & 60 & 57 & & \\
\hline \multicolumn{12}{|c|}{ Ear to Ear (in cm) } \\
\hline Overall & 100 & 17.81 & 0.84 & 15.5 & 17 & 18 & 18.5 & 19.5 & 18 & & \\
\hline
\end{tabular}




\begin{tabular}{|c|c|c|c|c|c|c|c|c|c|c|c|}
\hline Females & 50 & 17.3 & 0.7 & 15.5 & 16.6 & 17.45 & 17.5 & 19 & 17.5 & \multirow{2}{*}{$\begin{array}{c}\text { Mann- } \\
\text { Whitney } \\
\text { test }\end{array}$} & \multirow{2}{*}{$<0.001$} \\
\hline Males & 50 & 18.3 & 0.6 & 17 & 18 & 18.5 & 19 & 19.5 & 18 & & \\
\hline \multicolumn{12}{|c|}{ Nasion to Inion (in cm) } \\
\hline Overall & 100 & 10.7 & 0.7 & 9.5 & 10.2 & 10.5 & 11 & 12 & 11 & & \\
\hline Females & 50 & 10.4 & 0.5 & 9.5 & 10 & 10.5 & 10.7 & 11.5 & 10.5 & \multirow{2}{*}{$\begin{array}{c}\text { Mann- } \\
\text { Whitney } \\
\text { test }\end{array}$} & \multirow{2}{*}{$<0.001$} \\
\hline Males & 50 & 11.1 & 0.7 & 9.5 & 10.5 & 11 & 11.5 & 12 & 11 & & \\
\hline
\end{tabular}

${ }^{\star}$ Since most of the data except height was non-parametric as per Shapiro-Wilk Test and D’Agostino-Pearson test for normality hence Mann Whitney Test mostly used while $t$ test used in height only

Data collection and analysis was done using Microsoft Excel with Xrealstats ${ }^{10}$ add-on, Epi info version 7.2.4.0 (CDC Atlanta) $)^{11}$ and Medcalc Statistical Software version $20.006^{12}$. Parametric and non-parametric methods were used especially Descriptive analysis and Spearman's rank Coefficient. The p-value (for two tailed analysis) less than 0.05 was considered significant and p-value less 0.01 was considered highly significant.

\section{Results}

The age of participants ranged from 18-21 years. The mean age of the participants was $18.64 \pm 0.77$ years (males: 18.74 \pm 0.88 years and females: $18.54 \pm 0.65$ years). The mean BMI was $21.8 \pm 3.3 \mathrm{~kg} / \mathrm{m}^{2}$ (males: $23.01 \pm 3.3 \mathrm{~kg} / \mathrm{m}^{2}$ and females: $20.71 \pm 2.9 \mathrm{~kg} / \mathrm{m}^{2}$ ) with a range $16.2-32.8 \mathrm{~kg} / \mathrm{m}^{2}$. The head circumference of the participants ranged from

Table 2. Descriptive statistics of BAER variables of the study participants

\begin{tabular}{|c|c|c|c|c|c|c|c|c|c|c|}
\hline Parameter & Side & Mean & Std Dev & Min & $25 \%$ & Median & $75 \%$ & Max & Mode & p-value ${ }^{\star}$ \\
\hline \multirow{2}{*}{ I (ms) } & Left & 1.66 & 0.18 & 1.17 & 1.52 & 1.65 & 1.79 & 2 & 1.65 & \multirow{2}{*}{$<0.0001$} \\
\hline & Right & 1.56 & 0.17 & 1.38 & 1.44 & 1.48 & 1.63 & 2.2 & 1.42 & \\
\hline \multirow{2}{*}{ II (ms) } & Left & 2.78 & 0.20 & 2.46 & 2.6 & 2.73 & 2.98 & 3.13 & 3.08 & \multirow{2}{*}{0.1846} \\
\hline & Right & 2.74 & 0.21 & 2.17 & 2.56 & 2.73 & 2.9 & 3.08 & 2.5 & \\
\hline \multirow{2}{*}{ III (ms) } & Left & 3.68 & 0.20 & 3.35 & 3.52 & 3.69 & 3.85 & 4.4 & 3.65 & \multirow{2}{*}{0.0001} \\
\hline & Right & 3.61 & 0.17 & 3.35 & 3.48 & 3.6 & 3.69 & 3.94 & 3.35 & \\
\hline \multirow{2}{*}{ IV (ms) } & Left & 4.91 & 0.22 & 4.5 & 4.73 & 5.02 & 5.08 & 5.27 & 5.08 & \multirow{2}{*}{0.3228} \\
\hline & Right & 4.90 & 0.18 & 4.49 & 4.73 & 4.94 & 5.08 & 5.29 & 5.08 & \\
\hline \multirow{2}{*}{$\mathrm{V}(\mathrm{ms})$} & Left & 5.61 & 0.35 & 5.21 & 5.35 & 5.56 & 5.73 & 6.92 & 5.21 & \multirow{2}{*}{0.0049} \\
\hline & Right & 5.53 & 0.35 & 5.21 & 5.27 & 5.48 & 5.65 & 6.94 & 5.21 & \\
\hline \multirow{2}{*}{$\begin{array}{c}\text { I-III IPL } \\
\text { difference (ms) }\end{array}$} & Left & 2.03 & 0.22 & 1.35 & 1.92 & 2.04 & 2.17 & 2.98 & 2.08 & \multirow{2}{*}{0.1083} \\
\hline & Right & 2.06 & 0.21 & 1.35 & 1.92 & 2.05 & 2.17 & 2.95 & 1.92 & \\
\hline \multirow{2}{*}{$\begin{array}{c}\text { I-V IPL } \\
\text { difference (ms) }\end{array}$} & Left & 3.95 & 0.37 & 3.21 & 3.71 & 3.88 & 4.1 & 5.27 & 3.88 & \multirow{2}{*}{0.2942} \\
\hline & Right & 3.97 & 0.36 & 3.21 & 3.75 & 3.91 & 4.08 & 5.46 & 3.79 & \\
\hline \multirow{2}{*}{$\begin{array}{c}\text { III-V IPL } \\
\text { difference (ms) }\end{array}$} & Left & 1.92 & 0.39 & 1.17 & 1.71 & 1.8 & 2.04 & 3.27 & 1.75 & \multirow{2}{*}{0.6192} \\
\hline & Right & 1.92 & 0.36 & 1.27 & 1.75 & 1.87 & 2.04 & 3.5 & 1.88 & \\
\hline \multirow{2}{*}{$\begin{array}{c}\text { I-Ia difference } \\
(\mathrm{mV})\end{array}$} & Left & 1.46 & 1.89 & 0.05 & 0.47 & 0.75 & 1.29 & 8.49 & 0.75 & \multirow{2}{*}{0.0366} \\
\hline & Right & 0.94 & 1.09 & 0.08 & 0.46 & 0.69 & 0.99 & 8.17 & 0.69 & \\
\hline \multirow{2}{*}{$\begin{array}{l}\text { V-Va difference } \\
(\mathbf{m V})\end{array}$} & Left & 1.98 & 2.45 & 0.12 & 0.77 & 1.21 & 1.5 & 13.6 & 1.27 & \multirow{2}{*}{0.0003} \\
\hline & Right & 1.21 & 1.27 & 0.26 & 0.79 & 0.99 & 1.25 & 9.64 & 1.02 & \\
\hline \multirow{2}{*}{$\begin{array}{l}\text { Amplitude } \\
\qquad(\mathbf{m V})\end{array}$} & Left & 1.98 & 2.10 & 0.03 & 0.98 & 1.46 & 2.10 & 15.8 & 1.12 & \multirow{2}{*}{0.7531} \\
\hline & Right & 1.80 & 1.59 & 0.05 & 1 & 1.45 & 2.18 & 12.8 & 1.80 & \\
\hline
\end{tabular}

${ }^{\star}$ Wilcoxon test (paired samples)- non parametric data 
52.5-60 cm with mean $55.5 \pm 1.8 \mathrm{~cm}$ (males: $56.5 \pm 1.4 \mathrm{~cm}$ and females: $54.5 \pm 1.5 \mathrm{~cm}$ ) (Table 1).

All the Anthropometric measures (Table 1) had statistically significant difference in male and females. Age did not show any statistically significant difference.

Table 2 shows variables of BAER documented for the Left and Right ears of the study participants. The difference between Left and Right ears was found to be significant for Wave I, III and V, besides I- $\mathrm{I}_{\mathrm{a}}$ and $\mathrm{V}-\mathrm{V}_{\mathrm{a}}$ difference. The rest of values of the BAER variables were comparable (non-significant) for Left and Right ear.
Tables 3 and 4 depict the comparison of various BAEP latencies in males and females for left and right ear respectively. It is evident from the results that for both ears, although all the peak latencies (except wave II and IV in left ear) and IPL were higher in males as compared to females while the amplitude was higher in females in both ears.

The present study shows that the differences between male and female peak latencies of Wave III and V of left ear; Wave III, IV and V of right ear, I-V IPL and V-Va of Right ear were found to be statistically significant.

Table 3. Descriptive statistics of BAER variables of left ear gender wise

\begin{tabular}{|c|c|c|c|c|c|c|c|c|c|c|}
\hline Parameter & Gender & Mean & Std Dev & Min & $25 \%$ & Median & $75 \%$ & $\operatorname{Max}$ & Mode & p-value ${ }^{*}$ \\
\hline \multirow{2}{*}{ I (ms) } & Female & 1.63 & 0.15 & 1.42 & 1.52 & 1.65 & 1.77 & 1.94 & 1.52 & \multirow{2}{*}{0.189} \\
\hline & Male & 1.68 & 0.20 & 1.17 & 1.52 & 1.65 & 1.85 & 2 & 1.65 & \\
\hline \multirow{2}{*}{ II (ms) } & Female & 2.78 & 0.20 & 2.46 & 2.6 & 2.73 & 2.98 & 3.08 & 3.08 & \multirow{2}{*}{0.866} \\
\hline & Male & 2.78 & 0.20 & 2.5 & 2.65 & 2.75 & 2.98 & 3.13 & 2.65 & \\
\hline \multirow{2}{*}{ III (ms) } & Female & 3.66 & 0.20 & 3.35 & 3.52 & 3.63 & 3.77 & 4.4 & 3.52 & \multirow{2}{*}{0.045} \\
\hline & Male & 3.71 & 0.20 & 3.35 & 3.6 & 3.77 & 3.85 & 4.02 & 3.85 & \\
\hline \multirow{2}{*}{ IV (ms) } & Female & 4.94 & 0.20 & 4.5 & 4.77 & 5.02 & 5.08 & 5.27 & 5.08 & \multirow{2}{*}{0.572} \\
\hline & Male & 4.88 & 0.23 & 4.5 & 4.65 & 4.96 & 5.08 & 5.21 & 5.08 & \\
\hline \multirow{2}{*}{$\mathrm{V}(\mathrm{ms})$} & Female & 5.56 & 0.40 & 5.21 & 5.31 & 5.44 & 5.65 & 6.92 & 5.21 & \multirow{2}{*}{0.005} \\
\hline & Male & 5.66 & 0.29 & 5.21 & 5.52 & 5.6 & 5.81 & 6.79 & 5.56 & \\
\hline \multirow{2}{*}{$\begin{array}{c}\text { I-III IPL } \\
\text { difference }(\mathrm{ms})\end{array}$} & Female & 2.02 & 0.23 & 1.54 & 1.88 & 2 & 2.13 & 2.98 & 1.96 & \multirow{2}{*}{0.488} \\
\hline & Male & 2.04 & 0.22 & 1.35 & 1.94 & 2.05 & 2.17 & 2.77 & 2.08 & \\
\hline \multirow{2}{*}{$\begin{array}{c}\text { I-V IPL } \\
\text { difference }(\mathrm{ms})\end{array}$} & Female & 3.93 & 0.40 & 3.42 & 3.69 & 3.83 & 4.02 & 5.27 & 4 & \multirow{2}{*}{0.102} \\
\hline & Male & 3.98 & 0.34 & 3.21 & 3.79 & 3.92 & 4.1 & 5.23 & 3.88 & \\
\hline \multirow{2}{*}{$\begin{array}{c}\text { III-V IPL } \\
\text { difference (ms) }\end{array}$} & Female & 1.90 & 0.43 & 1.17 & 1.71 & 1.79 & 1.92 & 3.27 & 1.75 & \multirow{2}{*}{0.325} \\
\hline & Male & 1.94 & 0.35 & 1.46 & 1.71 & 1.865 & 2.13 & 3.15 & 1.75 & \\
\hline \multirow{2}{*}{$\begin{array}{c}\text { I-Ia difference } \\
(\mathrm{mV})\end{array}$} & Female & 1.34 & 1.69 & 0.05 & 0.68 & 0.78 & 1.12 & 8.49 & 0.73 & \multirow{2}{*}{0.275} \\
\hline & Male & 1.57 & 2.09 & 0.15 & 0.41 & 0.655 & 1.52 & 8.32 & 0.15 & \\
\hline \multirow{2}{*}{$\begin{array}{c}\text { V-Va difference } \\
(\mathrm{mV})\end{array}$} & Female & 1.90 & 2.35 & 0.58 & 1 & 1.25 & 1.49 & 13.55 & 1.27 & \multirow{2}{*}{0.176} \\
\hline & Male & 2.07 & 2.56 & 0.12 & 0.63 & 1.10 & 1.51 & 10.18 & 1.38 & \\
\hline \multirow{2}{*}{$\begin{array}{l}\text { Amplitude } \\
(\mathrm{mV})\end{array}$} & Female & 2.18 & 2.61 & 0.3 & 1.06 & 1.53 & 1.94 & 15.84 & 1.55 & \multirow{2}{*}{0.457} \\
\hline & Male & 1.79 & 1.41 & 0.03 & 0.97 & 1.30 & 2.32 & 8.18 & 1.12 & \\
\hline
\end{tabular}

Table 4. Descriptive statistics of BAER variables of right ear gender wise

\begin{tabular}{|c|c|c|c|c|c|c|c|c|c|c|}
\hline Parameter & Gender & Mean & Std Dev & Min & $\mathbf{2 5 \%}$ & Median & $\mathbf{7 5 \%}$ & Max & Mode & p-value \\
\hline \multirow{2}{*}{ I (ms) } & Female & 1.54 & 0.15 & 1.38 & 1.42 & 1.48 & 1.6 & 2.15 & 1.42 & \multirow{2}{*}{0.624} \\
\cline { 2 - 12 } & Male & 1.57 & 0.18 & 1.4 & 1.44 & 1.48 & 1.69 & 2 & 1.42 & 2.42 \\
\hline \multirow{2}{*}{ II (ms) } & Female & 2.72 & 0.20 & 2.33 & 2.52 & 2.71 & 2.85 & 3.08 & 2.52 & 2.382 \\
\cline { 2 - 11 } & Male & 2.76 & 0.21 & 2.17 & 2.6 & 2.73 & 2.94 & 3.08 & 2.5 & \\
\hline
\end{tabular}




\begin{tabular}{|c|c|c|c|c|c|c|c|c|c|c|}
\hline \multirow{2}{*}{ III (ms) } & Female & 3.56 & 0.15 & 3.35 & 3.44 & 3.56 & 3.65 & 3.85 & 3.35 & \multirow{2}{*}{0.0016} \\
\hline & Male & 3.67 & 0.18 & 3.35 & 3.56 & 3.65 & 3.81 & 3.94 & 3.35 & \\
\hline \multirow{2}{*}{ IV (ms) } & Female & 4.87 & 0.19 & 4.6 & 4.69 & 4.85 & 5.06 & 5.29 & 5.08 & \multirow{2}{*}{0.0484} \\
\hline & Male & 4.93 & 0.18 & 4.49 & 4.77 & 4.98 & 5.08 & 5.13 & 5.08 & \\
\hline \multirow{2}{*}{ V (ms) } & Female & 5.48 & 0.39 & 5.21 & 5.21 & 5.35 & 5.52 & 6.85 & 5.21 & \multirow{2}{*}{0.0036} \\
\hline & Male & 5.58 & 0.31 & 5.21 & 5.4 & 5.52 & 5.73 & 6.94 & 5.52 & \\
\hline \multirow{2}{*}{$\begin{array}{c}\text { I-III IPL } \\
\text { difference } \\
(\mathrm{ms})\end{array}$} & Female & 2.02 & 0.18 & 1.35 & 1.92 & 2 & 2.13 & 2.44 & 1.92 & \multirow[b]{2}{*}{0.058} \\
\hline & Male & 2.11 & 0.23 & 1.67 & 1.94 & 2.09 & 2.21 & 2.95 & 1.92 & \\
\hline \multirow{2}{*}{$\begin{array}{c}\text { I-V IPL } \\
\text { difference } \\
(\mathrm{ms})\end{array}$} & Female & 3.94 & 0.39 & 3.21 & 3.73 & 3.81 & 4.04 & 5.25 & 3.79 & \multirow[b]{2}{*}{0.0347} \\
\hline & Male & 4.01 & 0.32 & 3.52 & 3.79 & 4 & 4.13 & 5.46 & 4 & \\
\hline \multirow{2}{*}{$\begin{array}{c}\text { III-V IPL } \\
\text { difference } \\
(\mathrm{ms})\end{array}$} & Female & 1.92 & 0.39 & 1.52 & 1.67 & 1.83 & 1.92 & 3.25 & 1.65 & \multirow[b]{2}{*}{0.097} \\
\hline & Male & 1.92 & 0.33 & 1.27 & 1.79 & 1.92 & 2.04 & 3.5 & 1.88 & \\
\hline \multirow{2}{*}{$\begin{array}{c}\text { I-Ia } \\
\text { difference } \\
(\mathrm{mV})\end{array}$} & Female & 0.93 & 0.76 & 0.1 & 0.46 & 0.8 & 1.04 & 4.67 & 0.79 & \multirow[b]{2}{*}{0.0598} \\
\hline & Male & 0.95 & 1.35 & 0.08 & 0.43 & 0.61 & 0.82 & 8.17 & 0.37 & \\
\hline \multirow{2}{*}{$\begin{array}{c}\text { V-Va } \\
\text { difference } \\
(\mathrm{mV})\end{array}$} & Female & 1.12 & 0.43 & 0.26 & 0.85 & 1.09 & 1.33 & 2.74 & 1.19 & \multirow[b]{2}{*}{0.016} \\
\hline & Male & 1.30 & 1.75 & 0.28 & 0.69 & 0.91 & 1.06 & 9.64 & 1.02 & \\
\hline \multirow{2}{*}{$\begin{array}{l}\text { Amplitude } \\
(\mathrm{mV})\end{array}$} & Female & 1.89 & 1.98 & 0.17 & 0.97 & 1.36 & 2.06 & 12.77 & 0.4 & \multirow{2}{*}{0.62} \\
\hline & Male & 1.71 & 1.07 & 0.05 & 1.05 & 1.51 & 2.28 & 5.42 & 1.42 & \\
\hline
\end{tabular}

IPL= Interpeak Latency; ${ }^{\star}$ Mann-Whitney U Test for Two Independent Samples -non parametric data

Tables 5 and 6 show the correlation between BAER variables and anthropometric measures of the present study. BMI and Ear to Ear measurement showed no correlation to any BAER variables.

Height shows a positive correlation for peak latency of Wave V and I-V IPL difference for left ear while with peak latencies of Wave II, III and IV of right ear.
Weight also had a positive correlation with peak latency of Wave V of left ear and peak latency of Wave III and I-III IPL difference of right ear.

In the present study Inion to Nasion (IN) distance showed a positive correlation with peak latency of Wave III and IV besides I-III and I-Ia IPL difference and Amplitude of Right ear.

Table 5. Spearman Coefficient Correlation between BAER variables with Height, Weight and BMI

\begin{tabular}{|c|c|c|c|c|c|c|c|}
\hline \multirow{2}{*}{ Parameter } & \multirow{2}{*}{$\begin{array}{c}\text { Spearman's } \\
\text { coefficient Test }\end{array}$} & \multicolumn{2}{|c|}{ Height* $^{*}$} & \multicolumn{2}{|c|}{ Weight } & \multicolumn{2}{|c|}{ BMI } \\
\cline { 3 - 8 } & rho & 0.135 & 0.0939 & 0.0284 & -0.0235 & -0.0438 & -0.0770 \\
\hline \multirow{3}{*}{ I (ms) } & p-value & 0.1796 & 0.3529 & 0.7790 & 0.8168 & 0.6653 & 0.4465 \\
\cline { 2 - 8 } & rho & -0.00151 & 0.133 & -0.0658 & 0.0750 & -0.0698 & 0.0208 \\
\hline \multirow{2}{*}{ II (ms) } & p-value & 0.9881 & 0.1884 & 0.5154 & 0.4581 & 0.4902 & 0.8370 \\
\cline { 2 - 8 } & rho & 0.179 & 0.239 & 0.0849 & 0.221 & -0.0155 & 0.126 \\
\hline \multirow{2}{*}{ III (ms) } & p-value & 0.0743 & $\mathbf{0 . 0 1 6 5}$ & 0.4012 & $\mathbf{0 . 0 2 6 9}$ & 0.8781 & 0.2109 \\
\cline { 2 - 8 } & rho & -0.0622 & 0.197 & -0.141 & 0.0959 & -0.173 & -0.00462 \\
\hline \multirow{2}{*}{ IV (ms) } & p-value & 0.5386 & $\mathbf{0 . 0 4 9 4}$ & 0.1615 & 0.3425 & 0.0844 & 0.9636 \\
\cline { 2 - 8 } & & & &
\end{tabular}




\begin{tabular}{|c|c|c|c|c|c|c|c|}
\hline \multirow{2}{*}{$\mathrm{V}(\mathrm{ms})$} & rho & 0.333 & 0.197 & 0.205 & 0.123 & 0.0229 & 0.0423 \\
\hline & p-value & 0.0007 & 0.0490 & 0.0403 & 0.2246 & 0.8210 & 0.6758 \\
\hline \multirow{2}{*}{$\begin{array}{c}\text { I-III IPL } \\
\text { difference }(\mathrm{ms})\end{array}$} & rho & 0.0396 & 0.153 & 0.104 & 0.224 & 0.101 & 0.181 \\
\hline & p-value & 0.6958 & 0.1283 & 0.3016 & 0.0253 & 0.3150 & 0.0708 \\
\hline \multirow{2}{*}{$\begin{array}{c}\text { I-V IPL } \\
\text { difference }(\mathrm{ms})\end{array}$} & rho & 0.213 & 0.130 & 0.131 & 0.104 & 0.00531 & 0.0517 \\
\hline & p-value & 0.0335 & 0.1974 & 0.1925 & 0.3047 & 0.9581 & 0.6097 \\
\hline \multirow{2}{*}{$\begin{array}{c}\text { III-V IPL } \\
\text { difference (ms) }\end{array}$} & rho & 0.166 & 0.0921 & 0.0823 & 0.0137 & -0.0154 & -0.0208 \\
\hline & p-value & 0.0995 & 0.3620 & 0.4157 & 0.8920 & 0.8794 & 0.8371 \\
\hline \multirow{2}{*}{$\begin{array}{c}\text { I-Ia difference } \\
(\mathrm{mV})\end{array}$} & rho & -0.153 & -0.160 & -0.174 & -0.138 & -0.107 & -0.00444 \\
\hline & p-value & 0.1293 & 0.1125 & 0.0830 & 0.1710 & 0.2914 & 0.9650 \\
\hline \multirow{2}{*}{$\begin{array}{c}\text { V-Va } \\
\text { difference } \\
(\mathrm{mV})\end{array}$} & rho & -0.148 & -0.146 & -0.108 & -0.106 & -0.0308 & -0.0307 \\
\hline & p-value & 0.1424 & 0.1476 & 0.2849 & 0.2921 & 0.7612 & 0.7621 \\
\hline \multirow{2}{*}{$\begin{array}{l}\text { Amplitude } \\
\text { (mV) }\end{array}$} & rho & -0.0364 & 0.104 & 0.0320 & 0.119 & 0.0719 & 0.0437 \\
\hline & p-value & 0.7192 & 0.3048 & 0.7520 & 0.2382 & 0.4770 & 0.6656 \\
\hline
\end{tabular}

$\mathrm{IPL}=$ Interpeak Latency; ${ }^{\star}$ Height alone was parametric data but BAER parameters were non-parametric in nature hence Spearman's rank coefficient test applied

Table 6. Spearman coefficient correlation between BAER variables with Ear to Ear, IN and Head Circumference

\begin{tabular}{|c|c|c|c|c|c|c|c|}
\hline \multirow{2}{*}{ Parameter } & \multirow{2}{*}{$\begin{array}{c}\text { Spearman's } \\
\text { coefficient Test }\end{array}$} & \multicolumn{2}{|c|}{ Ear to Ear } & \multicolumn{2}{|c|}{ IN } & \multicolumn{2}{|c|}{ Head Circumference } \\
\hline & & Left & Right & Left & Right & Left & Right \\
\hline \multirow{2}{*}{ I (ms) } & rho & -0.00912 & 0.0253 & 0.00438 & 0.0728 & 0.0644 & -0.0134 \\
\hline & p-value & 0.9283 & 0.8029 & 0.9655 & 0.4715 & 0.5245 & 0.8946 \\
\hline \multirow{2}{*}{ II (ms) } & rho & -0.117 & 0.0777 & 0.110 & 0.117 & -0.0284 & 0.0799 \\
\hline & p-value & 0.2457 & 0.4424 & 0.2748 & 0.2459 & 0.7788 & 0.4297 \\
\hline \multirow{2}{*}{ III (ms) } & rho & 0.0418 & 0.168 & 0.105 & 0.321 & 0.145 & 0.218 \\
\hline & p-value & 0.6794 & 0.0953 & 0.2963 & 0.0011 & 0.1498 & 0.0295 \\
\hline \multirow{2}{*}{ IV (ms) } & rho & -0.114 & 0.137 & -0.179 & 0.218 & -0.132 & 0.215 \\
\hline & p-value & 0.2597 & 0.1756 & 0.0743 & 0.0295 & 0.1906 & 0.0318 \\
\hline \multirow{2}{*}{$\mathrm{V}(\mathbf{m s})$} & rho & 0.161 & 0.0385 & 0.161 & 0.110 & 0.325 & 0.178 \\
\hline & p-value & 0.1104 & 0.7038 & 0.1091 & 0.2755 & 0.0010 & 0.0757 \\
\hline \multirow{2}{*}{$\begin{array}{c}\text { I-III IPL } \\
\text { difference (ms) }\end{array}$} & rho & 0.0628 & 0.129 & 0.0916 & 0.213 & 0.0928 & 0.192 \\
\hline & p-value & 0.5350 & 0.2017 & 0.3645 & 0.0331 & 0.3587 & 0.0559 \\
\hline \multirow{2}{*}{$\begin{array}{c}\text { I-V IPL } \\
\text { difference }(\mathrm{ms})\end{array}$} & rho & 0.138 & -0.0108 & 0.141 & 0.00910 & 0.273 & 0.119 \\
\hline & p-value & 0.1719 & 0.9153 & 0.1608 & 0.9284 & 0.0060 & 0.2368 \\
\hline \multirow{2}{*}{$\begin{array}{c}\text { III-V IPL } \\
\text { difference }(\mathrm{ms})\end{array}$} & rho & 0.176 & -0.0159 & 0.0687 & -0.0245 & 0.176 & 0.0599 \\
\hline & p-value & 0.0805 & 0.8756 & 0.4971 & 0.8091 & 0.0805 & 0.5536 \\
\hline \multirow{2}{*}{$\begin{array}{c}\text { I-Ia difference } \\
(\mathrm{mV})\end{array}$} & rho & -0.0764 & -0.0587 & -0.116 & -0.221 & -0.116 & -0.114 \\
\hline & p-value & 0.4502 & 0.5617 & 0.2494 & 0.0273 & 0.2517 & 0.2572 \\
\hline \multirow{2}{*}{$\begin{array}{c}\text { V-Va } \\
\text { difference } \\
(\mathrm{mV})\end{array}$} & rho & -0.0737 & -0.0087 & -0.130 & 0.00983 & -0.143 & -0.139 \\
\hline & p-value & 0.4664 & 0.9314 & 0.1978 & 0.9227 & 0.1553 & 0.1665 \\
\hline \multirow{2}{*}{$\begin{array}{l}\text { Amplitude } \\
(\mathrm{mV})\end{array}$} & rho & -0.0272 & 0.108 & 0.0130 & 0.264 & -0.106 & 0.0497 \\
\hline & p-value & 0.7885 & 0.2870 & 0.8980 & 0.0080 & 0.2934 & 0.6233 \\
\hline
\end{tabular}

IPL= Interpeak Latency 
Head circumference measurements also had positive correlation with peak latency of Wave V of Left ear, III and IV of Right ear along with I-V IPL difference of Left ear.

\section{Discussion}

The present pilot study was conducted to study the influence of gender and anthropometric parameters on the variables of brainstem auditory evoked potentials in audiometrically normal young adults.

\subsection{BAER Parameters}

\subsubsection{Wave Peak Latency}

The electrophysiological activity of the VIII ${ }^{\text {th }}$ cranial nerve i.e., the cochlear nerve and nuclei is reflected by wave 1; wave III of superior olivary nucleus and wave $\mathrm{V}$ of inferior colliculi. The present study wave peak latencies are comparable to studies of Chalak et al ${ }^{13}$ Jiang et $a l^{14}$, Gupta $e t a l^{15}$ and Hall et $a l^{16}$ but relatively more than Amaral et $a l^{17}$ as shown in Table 8 .

The present study found statistically significant differences between the peak latencies of Left and Right ear in wave I, III and V. (Table 2) Similar significant difference in latencies in right and left side in wave III was documented by Santos et al. ${ }^{18}$.

\subsubsection{Interpeak Latency (IPL)}

The commonest Interpeak latencies (IPLs) employed in clinical practice are I-V, I-III and III-V.

IPL between I-III, referred to the conduction in the brainstem auditory system between the eighth nerve closed to the cochlea and the lower pons and III-V conduction between the lower pons and the midbrain; while I-V the conduction from Cochlea to midbrain.

The present study wave peak latencies are comparable to studies of Chalak et al..$^{13}$ Jiang et al. ${ }^{14}$, Gupta et al ${ }^{15}$, Hall et al. ${ }^{16}$ and Amaral et al. ${ }^{17}$ as shown in Table 8.

There was a significant difference I-V IPL and $\mathrm{V}-\mathrm{V}_{\mathrm{a}}$ difference of the right ear between male and females.

\subsubsection{V/I Ratio}

The present study found V/I ratio had significant difference between left and right side. But no significant difference was there between males and females of respective ear (Table 7 ).

The mean amplitude ratio between wave $\mathrm{V}$ and $\mathrm{I}$ was not consistent with findings in adults by Gathe et al. ${ }^{19}$ and Ghugare et al. ${ }^{20}$, but the difference between right and left ear was also reported in control group by Thakur et al. ${ }^{21}$.

Inter study variations documented within side the BAER values may be attributed to variable cohort and sample sizes in distinctive different study populations from distinctive different geographical areas ${ }^{20}$.

\subsection{Gender}

The present study reported prolongation of latencies and IPL in males in most of BAEPs but statistically significant were only in peak latencies of Wave III and V of left ear; Wave III, IV and V of right ear, I-V IPL and V-Va of Right ear.

Similar findings were reported in many studies. Aoyagi et al $^{22}$ reported significantly shorter wave III and $\mathrm{V}$ along with I-III and I-V IPL in females as compared to males.

Table 7. Mean Wave V/I Amplitude ratio in subjects

\begin{tabular}{|c|c|c|c|c|c|c|c|c|c|}
\hline Parameters & Mean & Std Dev & Min & $25 \%$ & Median & $75 \%$ & $\operatorname{Max}$ & Mode & $\begin{array}{c}\text { p- } \\
\text { value }^{*}\end{array}$ \\
\hline \multicolumn{10}{|l|}{ V/I ratio Left Ear } \\
\hline Overall $^{* *}$ & 3.42 & 0.40 & 2.61 & 3.16 & 3.37 & 3.62 & 4.68 & 3.22 & \\
\hline Female & 3.43 & 0.35 & 2.87 & 3.19 & 3.39 & 3.62 & 4.34 & 3.22 & \multirow[t]{2}{*}{$>0.05$} \\
\hline Male & 3.41 & 0.44 & 2.61 & 3.14 & 3.35 & 3.68 & 4.68 & 3.14 & \\
\hline \multicolumn{10}{|l|}{ V/I ratio Right Ear } \\
\hline Overall $^{\star *}$ & 3.58 & 0.37 & 2.60 & 3.34 & 3.62 & 3.83 & 4.69 & 3.62 & \\
\hline Female & 3.58 & 0.35 & 2.60 & 3.38 & 3.62 & 3.74 & 4.65 & 3.62 & \multirow[t]{2}{*}{$>0.05$} \\
\hline Male & 3.59 & 0.39 & 2.76 & 3.34 & 3.62 & 3.84 & 4.69 & 3.83 & \\
\hline
\end{tabular}

${ }^{\star}$ Mann-Whitney U Test for Two Independent Samples -non parametric data; ${ }^{* *} \mathrm{~V} / \mathrm{I}$ ratio of Left and Right ear difference was significantly significant (overall, in females and males separately)-Wilcoxon test (paired samples) 
Table 8. Mean \pm SD comparison of BAER Parameters in different studies

\begin{tabular}{|c|c|c|c|c|c|c|c|}
\hline BAER Parameter & Side & Present Study & Chalak et $a l^{13}$ & Jiang et $a l^{14}$ & Gupta et $a l^{15}$ & Hall et al ${ }^{16}$ & Amaral et $a l^{17}$ \\
\hline \multirow{2}{*}{ I (ms) } & Left & $1.66 \pm 0.18$ & $1.66 \pm 0.22$ & $1.71 \pm 0.11$ & $1.68 \pm 0.12$ & $1.65 \pm 0.14$ & $1.5 \pm 0.15$ \\
\hline & Right & $1.56 \pm 0.17$ & $1.66 \pm 0.23$ & & & & \\
\hline \multirow{2}{*}{ III (ms) } & Left & $3.68 \pm 0.20$ & $3.68 \pm 0.18$ & $3.80 \pm 0.15$ & $3.70 \pm 0.14$ & $3.8 \pm 0.18$ & $3.57 \pm 0.18$ \\
\hline & Right & $3.61 \pm 0.17$ & $3.65 \pm 0.39$ & & & & \\
\hline \multirow{2}{*}{$\mathrm{V}(\mathbf{m s})$} & Left & $5.61 \pm 0.35$ & $5.64 \pm 0.29$ & $5.64 \pm 0.18$ & $5.56 \pm 0.12$ & $5.64 \pm 0.23$ & $5.53 \pm 0.21$ \\
\hline & Right & $5.53 \pm 0.35$ & $5.59 \pm 0.71$ & & & & \\
\hline \multirow{2}{*}{$\begin{array}{c}\text { I-III IPL } \\
\text { difference (ms) }\end{array}$} & Left & $2.03 \pm 0.22$ & $2.02 \pm 0.23$ & $2.09 \pm 0.11$ & $2.05 \pm 0.18$ & $2.15 \pm 0.14$ & $2.06 \pm 0.19$ \\
\hline & Right & $2.06 \pm 0.21$ & $2.04 \pm 0.26$ & & & & \\
\hline \multirow{2}{*}{$\begin{array}{c}\text { I-V IPL } \\
\text { difference (ms) }\end{array}$} & Left & $3.93 \pm 0.37$ & $3.92 \pm 0.48$ & $3.94 \pm 0.18$ & $3.91 \pm 0.16$ & $3.99 \pm 0.2$ & $3.98 \pm 0.23$ \\
\hline & Right & $3.98 \pm 0.36$ & $4.03 \pm 0.35$ & & & & \\
\hline \multirow{2}{*}{$\begin{array}{l}\text { III-V difference } \\
(\mathrm{mV})\end{array}$} & Left & $1.90 \pm 0.39$ & $2.02 \pm 0.49$ & $1.84 \pm 0.18$ & $1.86 \pm 0.14$ & $1.84 \pm 0.14$ & $1.79 \pm 0.25$ \\
\hline & Right & $1.94 \pm 0.36$ & $1.98 \pm 0.36$ & & & & \\
\hline
\end{tabular}

Similar results were echoed by Gupta et al. ${ }^{23}$, Soares et al. ${ }^{17}$ and Harinder et al. ${ }^{24}$. Maurizi et al. ${ }^{25}$ reported statistically significant difference between boys and girls in wave III and V latency values and in III-V and I-V intervals below 2 years and seemed to increase with age.

A similar I-V IPL difference was reported by Stockard et al. ${ }^{26}$. Solanki et al. ${ }^{27}$ found significant difference in IPL I-III and III-V but not for I-V.

In general, for BAEP latency, amplitude and IPL differences in late components of waveforms are observed more consistently ${ }^{4,25}$. A study by Tandon et al..$^{28}$ showed no sex related differences in 3-13 years age group while Harinder et al. ${ }^{24}$ showed significant differences between sexes in more than 15 years age group in IPL besides latencies. Gupta et al. ${ }^{29}$ reported statistical insignificance of BAEP latency differences among age matched elderly male and female subjects and excluded gender as a basis of variation in BAEP among elderly age group.

Inspite many studies the age of onset for sex-related difference to begin is uncertain ${ }^{4}$ and to what extent these differences exist are unknown.

Many Authors like Aoyagi et al. ${ }^{22}$ and Nikiforidis et $a l .^{30}$, have tried to explain these differences due to differences in head sizes but other authors like Trune et al. ${ }^{31}$ documented males had longer latencies than females with comparable head diameter, suggesting that factors other than head size are differentiating them and Durrant et al. $^{32}$ suggested extent to which relationships exist between head dimensions and ABR latency measures, this nonpathological variable may be completely neutralized through the use of interpeak latency ratios (e.g., wave $\mathrm{V}$ latency divided by wave I latency).

Evidences from recent studies have documented difference of cortical and subcortical processing due to gender making explanation of role of gender more complex.

\subsection{Anthropometric Measurements}

\subsubsection{Head size}

The head size taking the 3 parameters of head circum ference, Inion to Nasion (IN) distance and Ear to Ear distance in males was significantly larger than females; (Table 1) this is being attributed to the normal physiological growth differentiation between the two at the given age group.

No correlation was found in ear-to-ear measurement with BAERs while positive correlation was found between Inion to Nasion (IN) distance and peak latencies wave III, wave IV, I-III IPL and I-Ia difference beside Amplitude of right ear only. Head circumference was found to have positive correlation with peal latency of wave III, IV of right ear and V of the left ear besides I-V IPL of left ear (Table 6).

Dempsey et al..$^{33}$ reported a strong positive correlation between head size and the I-V interpeak interval in healthy individuals. Ghugare et al. ${ }^{20}$ also reported positive correlation between head size and V wave latency and a weaker positive correlation between head size and AEP interpeak latencies (IPLs) I-V and III-V. 
A positive correlation between with head circum ference reinforce the fact that it reflects brain size, which affects the conduction time of neural pathway, thus should be considered an independent variable while interpreting results ${ }^{20,33}$ but IPL have a stronger correlation with brain stem size than Head circumference ${ }^{34}$. Durrant et al. ${ }^{32}$ concluded IPL has no clinical relevance in their study where the weak correlation with I-V IPL was further lost in large samples.

\subsubsection{BMI}

The present study documented no significant correlation was found between various BAER variables with BMI. Although components of BMI i.e., weight (in wave V left ear and wave III and I-III IPL in right ear) and height (in wave V and I-V IPL in left ear and wave III, IV and $\mathrm{V}$ in right ear) showed positive correlations with some parameters of BAER.

The study concurred with authors like Ghugare et $a l^{20}$ that BMI showed no significant correlation with BAER variables; but varied with others like observations of Subramaniam et al. ${ }^{35}$ who reported an increase in the absolute latencies of waves I, III, and V obese adults with non-significant difference between interpeak latencies; Gupta et al. ${ }^{36}$ reported prolongation of BAEP absolute latencies III and V and interpeak latencies I-III and I-V (for both ears) in obese patients and Solanki et al. ${ }^{27}$ in their study emphasized BMI as an important variable influencing BAEP records.

The variance of present study to the above studies may be attributed to duration and type of obesity. Gupta et al. ${ }^{36}$ attributed the alteration in myelination in obese persons resulting in altered transmission and delay in latencies resulting in the said changes. In the present study only $16 \%$ were overweight and $2 \%$ were obese, hence the variance in results due to small sample representation is likely.

\section{Limitation of Study}

The relatively small sample size and regional nature of the data limits the generalization of study results to different geographical population. The sample representation of sub grouping e.g., age grouping and BMI is inadequate to come to conclusions. Also, the study does not take in account the influence of factors which are known to affect hearing e.g., diseases (like diabetes mellitus ${ }^{37}$ ) and personal habits (like smoking and alcohol use ${ }^{38,39}$ ).

\section{Conclusion}

This study was an exploratory pilot study only on healthy controls. Being a pilot study sample size is small, but provides us baseline data for future studies which will be conducted for establishment of baseline values.

AEP results are definitely affected by gender, with latency duration more among males than females in most BAERs but were significant in few.

The head circumference of a person appears to affect the BAER; although further studies with larger age and head circumference ranges are required for a definitive conclusion.

BMI in the study was not significant with BAER variables; further studies are advised with proper representation of sub groups of BMI with duration of Obesity.

\section{Contribution of Authors}

Dr. Anupinder Thind, Assistant professor, contributed in study design, data collection, analysis and preparation; Dr. Ravdeep Singh Assistant Professor and Dr. Avnish Kumar Professor and Head conceptualized the idea, worked on discussions, conclusions, and reviewed manuscript and Dr. Puneet Gambhir, Assistant Professor helped in analysis and final manuscript preparation.

\section{References}

1. Legatt AD. Brainstem Auditory Evoked Potentials (BAEPs). In: Aminoff MJ, Daroff RB, editors. Encyclopedia of the Neurological Sciences (Second Edition). Oxford: Academic Press; 2014. p. 505-8. https://doi.org/10.1016/B978-0-12385157-4.00520-0

2. Jewett DL, Romano MN, Williston JS. Human auditory evoked potentials: Possible brain stem components detected on the scalp. Science. 1970; 167(3924):1517-8. https://doi. org/10.1126/science.167.3924.1517. PMid:5415287

3. McFadden D, Martin GK, Stagner BB, Maloney MM. Sex differences in distortion-product and transient-evoked otoacoustic emissions compared. Journal of the Acoustical Society of America. 2009; 125(1):23946. https://doi.org/10.1121/1.3037231. PMid:19173411. PMCid:PMC2649658 
4. Solanki JD, Mehta HB. Sex as a source of variance affecting auditory evoked potential. The Egyptian Journal of Otolaryngology. 2015; 31(2):111-4. https://doi. org/10.4103/1012-5574.156094

5. Celesia G. Brainstem auditory evoked responses. Handbook of Clinical Neurophysiology: Elsevier; 2013. https://doi. org/10.1016/B978-0-7020-5310-8.00007-7

6. Krishnan A. Frequency-following response. Burkard RF EJ, Don M, editors, editor. Philadelphia: Lippincott Williams and Wilkins; 2007.

7. Cambridge ED. Young adult: Cambridge University Press; 2021

8. Misra U. Clinical Neurophysiology. 2nd ed. India: Elsevier; 2012.

9. GK Pal. Textbook of practical physiology. 2nd ed. Chennai: Oriental Longman; 2005.

10. Zaiontz C. Real statistics using excel real statistics resource pack software (Release 7.6) [Internet]. 2020. Available from: www.real-statistics.com.

11. Dean AG, Sunki GG, Friedman R, Lantinga M, Sangam S, Zubieta JC, et al. Epi Info ${ }^{\mathrm{Tm}}$, a database and statistics program for public health professionals. CDC, Atlanta, GA, USA; 2011.

12. MedCalc ${ }^{\oplus}$. Statistical Software version 20.006 (MedCalc Software Ltd, Ostend, Belgium [Internet]. 2021. Available from: www.medcalc.org.

13. Chalak S, Kale A, Deshpande VK, Biswas DA. Establishment of normative data for monaural recordings of auditory brainstem response and its application in screening patients with hearing loss. Journal of Clinical and Diagnostic Research. 2015; 7(12):2677-9.

14. Jiang Z, Zheng M, Sun D, Liu X. Brainstem auditory evoked responses from birth to adulthood: Normative data of latency and interval. Hearing Research. 1991; 54:67-74. https://doi.org/10.1016/0378-5955(91)90137-X

15. Gupta D, Vishwakarma SK. Brain stem auditory evoked response - A study in parameters. Indian Journal of Otolaryngology. 1989; 41(1):6-8. https://doi.org/10.1007/ BF02994030

16. Hall JI. New Handbook of Auditory Evoked Responses. Boston: Allyn \& Bacon/Longman; 2007.

17. Soares Id, Menezes Pd, Tenorio ATL, Pereira. Brainstem auditory evoked standardization potential of using a new Device Pro Fono. Journal of Scientific Update. 2010; 22(4):421-26. https://doi.org/10.1590/S010456872010000400010. PMid:21271093

18. Santos AS, Júnior NC. Brainstem evoked response in bus drivers with noise-induced hearing loss. Brazilian J Otorhinolaryngology. 2009; 75(5):753-9. https://doi. org/10.1590/S1808-86942009000500023. PMid:19893947
19. Gathe BM, Gandhe MB, Gandhe SM, Puttewar AN, Saraf C, Singh R. Brainstem Auditory Evoked Potentials (BAEP)- A pilot study conducted on young healthy adults from Central India. Journal of Clinical and Diagnostic Research. 2014; 8(6):Bc16-8. https://doi. org/10.7860/JCDR/2014/8720.4515. PMid:25120971 PMCid:PMC4129266

20. Ghugare BW, Jain S, Parmar DJ, Dinkar MR, Ninama R. Influence of BMI and head circumference on variables of auditory evoked potential in young healthy male human participants. The Egyptian Journal of Otolaryngology. 2016; 32(1):53-6. https://doi.org/10.4103/1012-5574.175854

21. Thakur L, Anand JP, Selvamurthy W. Brainstem auditory evoked responses in young urban and rural boys--a comparison. Journal of Environmental Biology. 2003; 24(3):321-5.

22. Aoyagi M, Kim Y, Yokoyama J, Kiren T, Suzuki Y, Koike Y. Head size as a basis of gender difference in the latency of the brainstem auditory-evoked response. Audiology. 1990; 29(2):107-12.https://doi.org/10.3109/00206099009081652. PMid:2350303

23. Gupta GGS, Singh PB, Singh NP, Kaiti R. Anthropometric differences and gender variations in brainstem auditory evoked responses - A cross-sectional study in North Indian population. Natl J Physiol Pharm Pharmacol. 2018; 8(1):6474. https://doi.org/10.5455/njppp.2018.8.0728231072017

24. Harinder JS, Sarup RS, Sharanjit K. The study of age and sex related changes in the auditory brainstem evoked potential. Journal of Clinical and Diagnostic Research. 2010; 4:34959.

25. Maurizi M, Ottaviani F, Paludetti G, Almadori G, Pierri F, Rosignoli M. Effects of sex on auditory brainstem responses in infancy and early childhood. Scandinavian Audiology. 1988; 17(3):143-6. https://doi. org/10.3109/14992028809042184. PMid:3206180

26. Stockard JE, Stockard JJ, Westmoreland BF, Corfits JL. Brainstem auditory-evoked responses: Normal variation as a function of stimulus and subject characteristics. Archives of Neurology. 1979; 36(13):823-31. https://doi.org/10.1001/ archneur.1979.00500490037006. PMid:508145

27. Solanki J, Joshi N, Mehta H, Shah C. A study of gender, head circumference and BMI as a variable affecting BAEP results of late teenagers. Indian Journal of Otology. 2012; 18(1):3-6. https://doi.org/10.4103/0971-7749.98276

28. Tandon OP. Auditory brainstem evoked responses in healthy north Indians. Indian Journal of Medical Research. 1990; 92:252-6.

29. Gupta S, Mittal S, Baweja P, Kumar A, Singh KD, Sharma R. Analysis of gender based differences in auditory evoked potentials among healthy elderly population. Advanced Biomedical Research. 2014; 3:208. https:// 
doi.org/10.4103/2277-9175.143243. PMid:25371865
PMCid:PMC4219204

0. Nikiforidis GC, Koutsojannis CM, Varakis JN, Goumas PD. Reduced variance in the latency and amplitude of the fifth wave of auditory brain stem response after normalization for head size. Ear and Hearing. 1993; 14(6):423-8. https://doi.org/10.1097/00003446-199312000-00008. PMid:8307247

31. Trune DR, Mitchell C, Phillips DS. The relative importance of head size, gender and age on the auditory brainstem response. Hearing Research. 1988; 32(2-3):165-74. https:// doi.org/10.1016/0378-5955(88)90088-3

32. Durrant JD, Sabo DL, Hyre RJ. Gender, head size, and ABRs examined in large clinical sample. Ear and Hearing. 1990; 11(3):210-4. https://doi.org/10.1097/00003446199006000-00008. PMid:2358132

33. Dempsey JJ, Censoprano E, Mazor M. Relationship between head size and latency of the auditory brainstem response. Audiology. 1986; 25(4-5):258-62. https://doi. org/10.3109/00206098609078391

34. Ponton CW, Eggermont JJ, Coupland SG, Winkelaar R. The relation between head size and auditory brainstem response interpeak latency maturation. Journal of the Acoustical Society of America. 1993; 94(4):2149-58. https://doi.org/10.1121/1.407486. PMid:8227754

35. Subramaniam A, Govindarajulu SN, Kumar S. A comparative study of auditory evoked potential in young obese and normal subjects. International Research Journal of Medicine and Medical Sciences. 2013; 1(8):11-4.

36. Gupta S, Gupta G, Kaiti R. Visual and brainstem auditory evoked potentials in obese and overweight individuals. National Journal of Physiology, Pharmacy and Pharmacology. 2017; 7(5):450-54. https://doi.org/10.5455/ njppp.2017.7.1130719122016

37. Mozaffari M, Tajik A, Ariaei N, Ali-Ehyaii F, Behnam H. Diabetes mellitus and sensorineural hearing loss among non-elderly people. The Eastern Mediterranean Health Journal. 2010; 16(9):947-52. https://doi. org/10.26719/2010.16.9.947. PMid:21218721

38. Zelman S. Correlation of smoking history with hearing loss. Jama. 1973; 223(8):920. https://doi.org/10.1001/ jama.1973.03220080050016. PMid:4739239

39. CRAWFORD A. Alcohol, auditory functioning and deafness. Addiction Biology. 1997; 2(2):125-50 https://doi. org/10.1080/13556219772688. PMid:26735631 